ARTICLE

Received 20 Mar 2016 | Accepted 21 Nov 2016 | Published 27 Jan $2017 \quad$ DOl: 10.1038/ncomms14000

\title{
Stimulated emission from nitrogen-vacancy centres in diamond
}

Jan Jeske ${ }^{1}$, Desmond W.M. Lau², Xavier Vidal ${ }^{3}$, Liam P. McGuinness ${ }^{4}$, Philipp Reineck ${ }^{2}$, Brett C. Johnson ${ }^{5}$, Marcus W. Doherty ${ }^{6}$, Jeffrey C. McCallum ${ }^{5}$, Shinobu Onoda ${ }^{7}$, Fedor Jelezko ${ }^{4}$, Takeshi Ohshima ${ }^{7}$, Thomas Volz ${ }^{3}$, Jared H. Cole', Brant C. Gibson ${ }^{2} \&$ Andrew D. Greentree ${ }^{1,2}$

Stimulated emission is the process fundamental to laser operation, thereby producing coherent photon output. Despite negatively charged nitrogen-vacancy $\left(\mathrm{NV}^{-}\right)$centres being discussed as a potential laser medium since the 1980s, there have been no definitive observations of stimulated emission from ensembles of $\mathrm{NV}^{-}$to date. Here we show both theoretical and experimental evidence for stimulated emission from $\mathrm{NV}^{-}$using light in the phonon sidebands around $700 \mathrm{~nm}$. Furthermore, we show the transition from stimulated emission to photoionization as the stimulating laser wavelength is reduced from 700 to $620 \mathrm{~nm}$. While lasing at the zero-phonon line is suppressed by ionization, our results open the possibility of diamond lasers based on $\mathrm{NV}^{-}$centres, tuneable over the phonon sideband. This broadens the applications of $\mathrm{NV}^{-}$magnetometers from single centre nanoscale sensors to a new generation of ultra-precise ensemble laser sensors, which exploit the contrast and signal amplification of a lasing system.

\footnotetext{
${ }^{1}$ Chemical and Quantum Physics, School of Science, RMIT University, Melbourne, Victoria 3001, Australia. ${ }^{2}$ ARC Centre of Excellence for Nanoscale BioPhotonics, School of Science, RMIT University, Melbourne, Victoria 3001, Australia. ${ }^{3}$ ARC Centre of Excellence for Engineered Quantum Systems, Department of Physics and Astronomy, Macquarie University, North Ryde Sydney, New South Wales 2109, Australia. ${ }^{4}$ Institut für Quantenoptik, Universität Ulm, Albert Einstein Allee 11, 89081 Ulm, Germany. ${ }^{5}$ School of Physics, University of Melbourne, Parkville, Melbourne, Victoria 3010, Australia. ${ }^{6}$ Laser Physics Centre, Research School of Physics and Engineering, Australian National University, Canberra, Australian Capital Territory 2601, Australia. ${ }^{7}$ National Institutes for Quantum and Radiological Science and Technology, Takasaki, Gunma 370-1292, Japan. Correspondence and requests for materials should be addressed to J.J. (email: janjeske@gmail.com or jan.jeske@rmit.edu.au).
} 
T he negatively charged nitrogen-vacancy centre in diamond ${ }^{1}$ has found wide applicability in quantum information $^{2-4}$ and sensing ${ }^{5}$ of magnetic fields ${ }^{6-11}$, electric fields ${ }^{12,13}$, temperature ${ }^{14,15}$, pressure $^{16}$ and biosensing ${ }^{17,18}$. For both readout and sensing, fluorescence that is, spontaneous emission is collected and the spin state is inferred from the different emission levels. The time-averaged spontaneous emission from the different spin states differs by no more than $30 \%$ and spontaneous emission is in all directions, creating the challenge of collecting the emission ${ }^{19}$.

Photons created by stimulated emission, on the other hand, have the same phase, wavelength and spatial mode as the stimulating light field. This enables close-to-unity collection efficiency as all photons are emitted into the same mode. Furthermore, the competition between spontaneous emission and stimulated emission potentially allows contrast of several orders of magnitude between the brightest and least bright state of the $\mathrm{NV}^{-}$centre compared with the typical $30 \%$ for single centres and $4 \%$ for ensembles under spontaneous emission.

Stimulated emission is also the basis for lasing, but despite the success of diamond Raman lasers ${ }^{20}$ and despite an early proposal for a laser based on diamond defects ${ }^{21}$, and the demonstration of cavity coupling ${ }^{10,22}$ a realization of an $\mathrm{NV}$ laser has not been demonstrated so far. Microscopy, based on stimulated emission depletion, a super-resolution technique taking advantage of emission reduction by a strong light field, has been successfully implemented with NV centres ${ }^{23}$ as well as NVN centres ${ }^{24}$. However, the photophysical process leading to said emission reduction, be it stimulated emission to the ground state, or photo- ionization to the charge neutral $\mathrm{NV}^{0}$ state ${ }^{25-27}$ has not been extensively studied in this context. Indeed, photo-ionization of the $\mathrm{NV}^{-} \leftrightarrow \mathrm{NV}^{0}$ has been shown to be induced by strong light pulses $^{28-31}$. The ionization process has been suggested to be a two-photon process ${ }^{26,28,29}$, which scales quadratically with laser power, for the wavelengths around the zero-phonon line (ZPL) of NV emission. Excited state absorption, for example via the ionization channel, can lead to weakening of stimulated emission by driving towards $\mathrm{NV}^{0}$. Insight into stimulated emission of $\mathrm{NV}$ centres is particularly important, since both ionization and stimulated emission by a strong light pulse result in reduced $\mathrm{NV}^{-}$spontaneous emission and are therefore hard to distinguish, and also owing to the multitude of applications benefiting from these competing processes.

Here we show experimentally that light with wavelengths above $650 \mathrm{~nm}$ does not induce net ionization of $\mathrm{NV}^{-}$centres, for powers up to a few Watts during the pulse, and show strong evidence for the presence of stimulated emission in the $\mathrm{NV}^{-}$ phonon side band. We use a green $(532 \mathrm{~nm})$ CW pump-laser together with a pulsed laser source with tunable wavelength to measure stimulated emission at different wavelengths. The pulses, with a duration of only $6 \mathrm{ps}$, provide an intense light field and create a measurable change in the emission properties. Since the emitted photons are indistinguishable from the laser pulse, we initially detect the stimulated emission indirectly by the reduction of spontaneous emission. In subsequent measurements, we demonstrate enhanced emission at the wavelength of the stimulating laser as direct evidence for stimulated emission. In addition, we measure the excited state population of both charge a

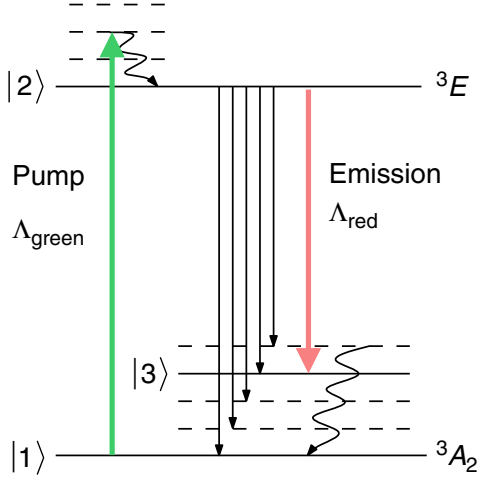

b

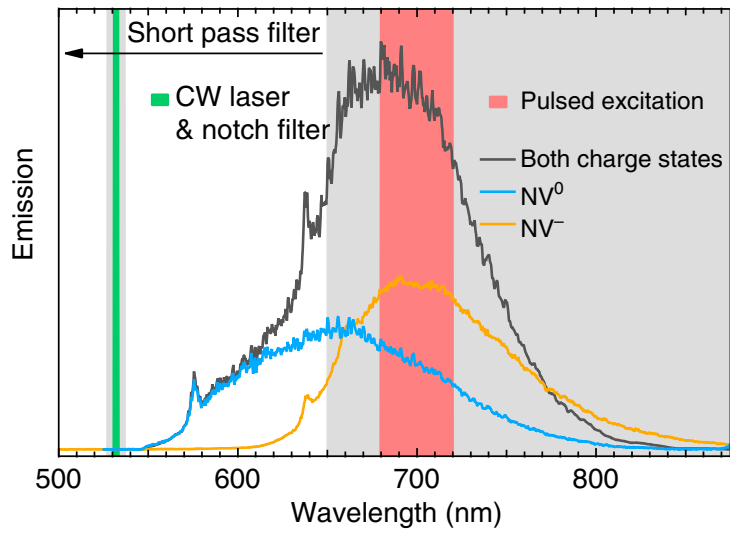

C

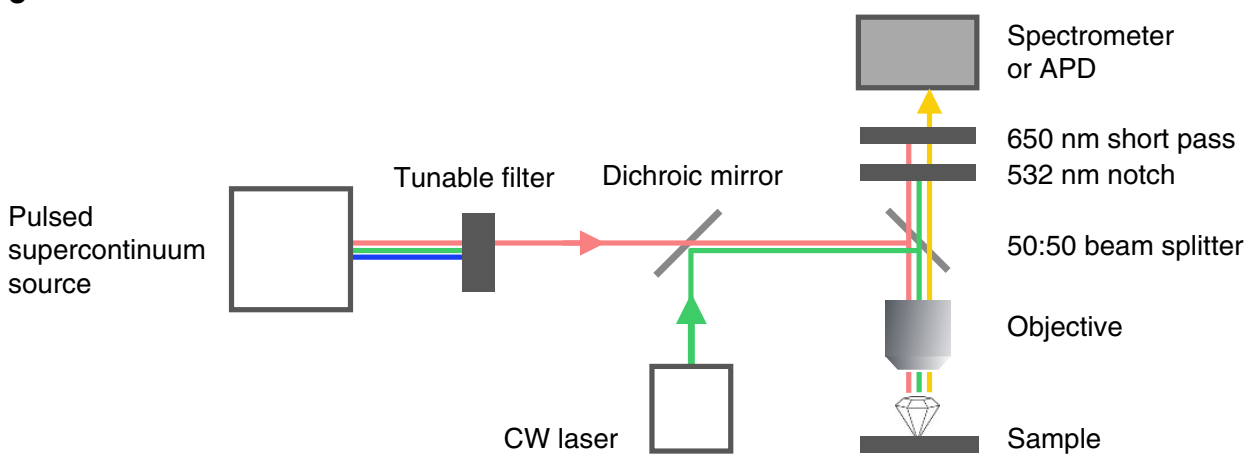

Figure 1 | Stimulated emission and experimental setup. (a) Nitrogen-vacancy centre ( $\mathrm{NV}^{-}$) level structure including phonon-added states (dashed and |3) ) with pumping and emission rates. Laser pumping (green) lifts population to the excited state, which can then decay via spontaneous emission (straight black arrows) or stimulated emission (red) due to interaction with a light field whose wavelength matches the $|2\rangle \leftrightarrow|3\rangle$ transition. (b) Laser excitation (red and green columns) with short pass and notch (grey). This setup blocks laser light from the detector but still allows to monitor both $\mathrm{NV}^{-}$and $\mathrm{NV}^{0}$ emission. Example spectra are included: $\mathrm{NV}^{-}$emission (orange), $\mathrm{NV}^{0}$ emission (blue) and mixed emission (grey). (c) Experimental setup. 
states separately by monitoring the corresponding ZPL intensities to detect any potential ionization. Our results show the presence of stimulated emission and the possibility of lasing with $\mathrm{NV}^{-}$ centres.

\section{Results}

Stimulated emission and experimental setup. Conventionally, both sensing and quantum state readout in the $\mathrm{NV}^{-}$centre is performed via fluorescence measurements after optical pumping, typically by exciting at a wavelength around $532 \mathrm{~nm}$, from the ${ }^{3} A_{2}$ ground state, which we denote $|1\rangle$, to the ${ }^{3} E$ excited state $|2\rangle$, see Fig. 1a. Spontaneous emission occurs at a rate $\approx(12 \mathrm{~ns})^{-1}$ over the characteristic $\mathrm{NV}^{-}$spectrum with $\mathrm{ZPL}$ at $637 \mathrm{~nm}$, corresponding to the $|1\rangle \leftrightarrow|2\rangle$ transition, and with phonon-sideband emission from 637 to $\sim 800 \mathrm{~nm}$, corresponding to transitions from $|2\rangle$ to short-lived vibronic states slightly above $|1\rangle$ with added phonons, followed by a rapid decay to $|1\rangle$ (refs 32,33 ).

Stimulated emission due to a coherent light field of wavelength $\lambda$ interacting with the $\mathrm{NV}$ centre occurs at a rate $\Lambda_{\text {red }}=\sigma_{\lambda} \mathrm{I} /$ $\left(\hbar \omega_{\lambda}\right)$ where $I$ is the power per area of the light field, $\hbar \omega_{\lambda}$ the corresponding photon energy and $\sigma_{\lambda}$ the stimulated emission cross-section, which as a function of $\lambda$ is proportional to the NV emission spectrum, that is, the spontaneous emission rate. Strong stimulated emission is achieved by strong fields, produced for example, in optical cavities or in our case by a pulsed supercontinuum source.

The rate of stimulated emission is increased for laser light in the phonon-sidebands' wavelengths because the phonon-sideband emission is stronger than the ZPL emission, therefore the crosssection for decay on the phononic sidebands is higher. Accordingly, we choose a pulsed laser excitation around $700 \mathrm{~nm}$ (red light) at the peak of the phonon sideband, Fig. 1b, to achieve strong stimulated emission at the same wavelength. This laser is resonant with the transition between the excited state $|2\rangle$ and a phonon-added ground state $|3\rangle$ and induces a stimulated emission rate, which competes with the spontaneous emission, Fig. 1a. The red laser will therefore enhance the emission probability around $700 \mathrm{~nm}$ and reduce the emission probability for the rest of the NV spectrum. For lasing, population inversion is also easier to obtain on the sideband transition since the population in $|3\rangle$ decays quickly and therefore it is no longer necessary to pump more than $50 \%$ of the NV centres out of the ground state $|1\rangle$. Our present setup, however, is not designed for self-sustained lasing because the light field does not build up in a cavity but is provided by the pulsed laser. The stimulated emission can therefore not be measured directly, since it is indistinguishable from the stronger pulsed laser signal. However the reduction in the rest of the NV emission spectrum can be detected. This reduction can be turned on and off with the red laser.

Obtaining full NV emission spectra is challenging when a strong laser at the centre of the $\mathrm{NV}$ emission spectrum $(\sim 700 \mathrm{~nm})$ is used. We used a $650 \mathrm{~nm}$ short pass and a $532 \mathrm{~nm}$ notch in front of the detector to block out the pulsed red laser and the green $(532 \mathrm{~nm}) \mathrm{CW}$ pump laser respectively before the light was detected with a spectrometer or avalanche photodiode detector (APD), Fig. 1b. This detection window allowed us to monitor both the $\mathrm{NV}^{-} \mathrm{ZPL}$ at $637 \mathrm{~nm}$ as well as the $\mathrm{NV}^{0} \mathrm{ZPL}$ at $575 \mathrm{~nm}$. Ionization is expected to increase the emission of one peak at the cost of the other and can be clearly distinguished in this setup from stimulated emission, which should decrease the emission in the entire monitored range of wavelengths. Even in the case that ionization $\mathrm{NV}^{-} \leftrightarrow \mathrm{NV}^{0}$ occurs both ways during the 6 ps pulse, the emission spectrum, which is mainly obtained in the 12.5 ns between pulses, should reflect the new relative charge state populations. The short pass optical filter allows us to vary the red laser wavelength, that is, measure stimulated emission as a function of wavelength.

We used a pulsed supercontinuum source (Fianium WL SC400-8) with a 6 ps pulse duration at a repetition rate of up to $80 \mathrm{MHz}$ with a tunable filter, Fig. 1c, which allowed us to select both the centre wavelength $(700 \mathrm{~nm}$ initially) and bandwidth ( $40 \mathrm{~nm}$ initially). The resulting time-averaged power of several $\mathrm{mW}$ corresponds to several Watts, during the pulse duration. The pulsed light was aligned via a dichroic mirror with the green $\mathrm{CW}$ laser and focused through an objective $(\mathrm{NA}=0.9)$ onto the NV diamond samples. We confirmed the effects on two $\sim 500 \mu \mathrm{m}$ thick single-crystal diamonds as well as a nanodiamond sample, all with very high $\mathrm{NV}$ density.

Emission reduction of both ZPLs. To investigate the effect of the pulsed laser on the spontanteous emission, we first measured the NV emission between 550 and $650 \mathrm{~nm}$ when only the green CW laser excites the sample. We then measured the spectra when both the green $\mathrm{CW}$ and the pulsed laser at 700 with $40 \mathrm{~nm}$ bandwidth are on. The data in Fig. 2a was taken over $100 \mathrm{~ms}$ acquisition time, which corresponds to 8 million pulses. The spectra are integrated over the entire acquisition time including the pulsed laser being on and off, and the spectra were smoothed with a running average over a $4 \mathrm{~nm}$ window. Figure $2 \mathrm{a}$ shows the emission with both the $\mathrm{NV}^{0} \mathrm{ZPL}$ at $575 \mathrm{~nm}$ and $\mathrm{NV}^{-} \mathrm{ZPL}$ at $637 \mathrm{~nm}$ clearly visible as expected since $532 \mathrm{~nm}$ pumping is known to excite both charge states of the NV. More importantly, the addition of the red pulsed laser reduces the entire emission in the observed wavelengths as expected when stimulated emission at $700 \mathrm{~nm}$ is induced. We note that the reduction occurs despite the fact that the red pulsed laser by itself creates weak excitation of the $\mathrm{NV}^{-}$as discussed later. Both $\mathrm{ZPLs}\left(\mathrm{NV}^{-}\right.$and $\left.\mathrm{NV}^{0}\right)$ are reduced, which indicates that the reduction of $\mathrm{NV}^{-}$emission is not caused by ionization towards $\mathrm{NV}^{0}$. If ionization to $\mathrm{NV}^{0}$ occurred, then we would expect the increased $\mathrm{NV}^{0}$ population to provide an increase of the $\mathrm{NV}^{0} \mathrm{ZPL}$ emission. Quite contrary, in Fig. 2a the $\mathrm{NV}^{0} \mathrm{ZPL}$ is also reduced indicating that the $\mathrm{NV}^{0}$ population also experiences stimulated emission at $700 \mathrm{~nm}$. While this might seem surprising, the phonon sideband of the $\mathrm{NV}^{0}$ extends over a slightly wider range than the $\mathrm{NV}^{-}$and is quite strong at $700 \mathrm{~nm}$, see Fig. $1 \mathrm{~b}$.

Our interpretation attributes the emission reduction in the observed wavelength range to stimulated emission at $700 \mathrm{~nm}$. As an initial check of this interpretation, we changed the pulsed laser wavelength to also be $532 \mathrm{~nm}$, as the CW source. This is outside the NV emission spectrum and stimulated emission should therefore not occur. Figure $2 \mathrm{~b}$ shows that in this case indeed the addition of the pulsed laser increases the total emission, proving that the effect of emission reduction by the added pulsed laser only occurs at certain wavelengths. For stimulated emission, this range of wavelengths would be expected to be the range of spontaneous emission, which we confirm further below.

How can the apparent occurrence of stimulated emission be brought into agreement with the established fact ${ }^{25-31}$ that strong light pulses around $637 \mathrm{~nm}$ efficiently decharge the $\mathrm{NV}^{-}$to the $\mathrm{NV}^{0}$ ? To explore the photoionization, we reduced the pulsed laser wavelength, swapping the $650 \mathrm{~nm}$ short pass for a $600 \mathrm{~nm}$ short pass and monitored the $\mathrm{NV}^{0} \mathrm{ZPL}$. Again, we combined a green $\mathrm{CW}$ laser, which creates $\mathrm{NV}^{0}$ emission, with the pulsed red light, which by itself does not create $\mathrm{NV}^{0}$ emission. Any change in the $\mathrm{NV}^{0}$ signal after adding pulsed light must therefore be due to either stimulated emission or ionization. Figure $2 \mathrm{c}$ shows clearly the transition between two regimes: For pulsed light at high wavelengths, $\lambda>680 \mathrm{~nm}$, the $\mathrm{NV}^{0}$ signal is reduced as discussed above and stimulated emission dominates. For pulsed light at lower wavelengths, $\lambda<640 \mathrm{~nm}$, the $\mathrm{NV}^{0}$ signal is increased and 
a

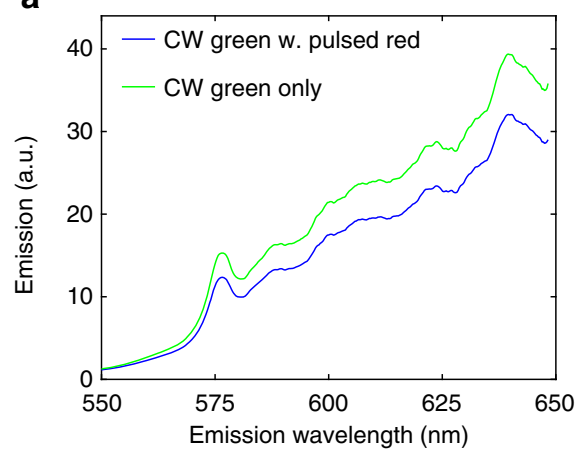

b

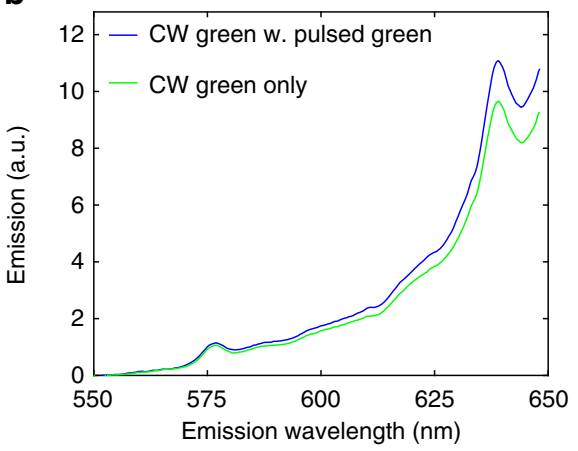

C

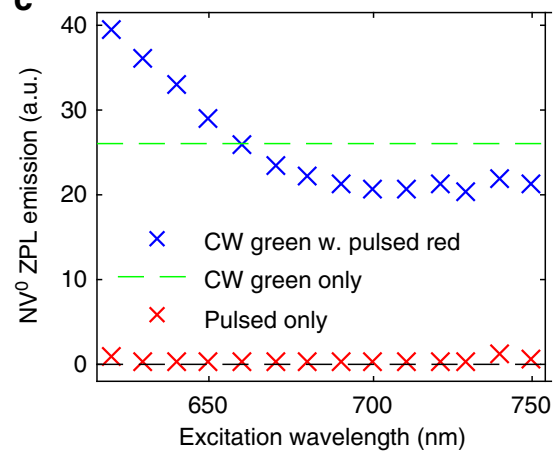

Figure 2 | Emission reduction of both zero-phonon lines. (a) Spectral emission of the NV centre ensemble due to $532 \mathrm{~nm}$ continuous wave (CW) laser excitation alone (green), and due to an added pulsed $700 \mathrm{~nm}$ laser excitation (blue). The emission with both lasers on is reduced compared with only green $\mathrm{CW}$ excitation. This is consistent with a preferential emission at $700 \mathrm{~nm}$ due to stimulated emission. (b) When the pulsed laser wavelength is changed to also be at $532 \mathrm{~nm}$, the emission is increased by the pulsed laser. All the spectra were smoothed with a $4 \mathrm{~nm}$ window. (c) Two regimes exist: the NV0 emission is decreased by the addition of pulsed laser wavelengths above $680 \mathrm{~nm}$, where stimulated emission dominates, and increased for pulsed wavelengths below $640 \mathrm{~nm}$, where ionization from $\mathrm{NV}^{-}$to $\mathrm{NV}^{0}$ is known to be the dominant process. In the measured range of wavelengths the pulsed laser alone does not create any $\mathrm{NV}^{0}$ signal, that is, there is no direct excitation. The pulsed power was kept constant at $2.9 \mathrm{~mW}$ for all excitation wavelengths.

ionization from $\mathrm{NV}^{-}$towards the $\mathrm{NV}^{0}$ is the dominant process $^{25}$. The threshold between the two behaviours seems to be around $660 \mathrm{~nm}$, that is, a photon energy of $1.88 \mathrm{eV}$. This energy value is slightly higher than theoretical calculations of the minimal energy value required for ionization from the $\mathrm{NV}^{-}$ excited state, which were predicted as $1.56 \mathrm{eV}$ in ref. 29 and 1.7 $\mathrm{eV}$ (private communication with Audrius Alkauskas). Note that this value is not to be confused with the ionization energy from the $\mathrm{NV}^{-}$ground state of $2.6 \mathrm{eV}$ (ref. 28).

The relative emission reduction at the $\mathrm{NV}^{-} \mathrm{ZPL}$ in Fig. $2 \mathrm{a}$ is about $18 \%$ and about $21 \%$ in the measurement of Fig. $2 \mathrm{c}$ for $700 \mathrm{~nm}$ pulsed wavelength. Assuming that the red intensity in the region of focus is intense enough to fully deplete the excited state via stimulated emission, this means that 18 to $21 \%$ of the $\mathrm{NV}$ centres that contribute to the signal are in the region of focus. Since we used a $500 \mu \mathrm{m}$ thick sample, the percentage value of focus volume relative to illuminated volume seems high but is realistic given that there is non-uniform intensity in all dimensions of the laser spot and considering that $\mathrm{NV}$ centres in the focus volume contribute more strongly to the emission signal.

Emission dynamics. While stimulated emission should only occur during the 6 ps pulse duration, a reduction by other indirect effects could show other timescales or no temporal structure. Changes in the emission due to ionization have been found to occur typically on much slower timescales of microseconds to milliseconds $25,26,30$

We therefore measured the total emission (not spectrally resolved) in the detected emission window (550 nm to $650 \mathrm{~nm}$, see Fig. 1b) with an APD and time-correlated counting card with a 0.1 ns temporal resolution. While the $6 \mathrm{ps}$ pulse is not resolved on that scale, the dynamics between pulses is resolved. The time between pulses was set to $25 \mathrm{~ns}$, corresponding to a repetition rate of $40 \mathrm{MHz}$. Figure 3a shows that the reduction due to the added pulsed laser (blue curve) relative to the green $\mathrm{CW}$ laser (green curve) occurs faster than the time resolution of the electronics and then the signal recovers between pulses due to the green CW pump, as expected. The emission due to the pulsed laser only (red curve) shows that the $700 \mathrm{~nm}$ pulse alone actually weakly excites the $\mathrm{NV}^{-}$centre. The time point of this excitation coincides with the reduction of the emission of the blue curve, that is, the time point of the pulse.

To further investigate the wavelength dependence, discovered in Fig. 2, we varied the centre wavelength of the pulsed laser in $20 \mathrm{~nm}$ steps. The full width at half maximum of the laser was also set to $20 \mathrm{~nm}$ to ensure no overlap between the steps. The strength of the reduction varies with the wavelength of the pulsed laser (Fig. 3b,c) and shows that the effect is the strongest at $700 \mathrm{~nm}$ and reduces towards $800 \mathrm{~nm}$. To ensure the data are independent of the small power variations of the source, which occurred when changing wavelengths, we measured the power for each wavelength and rescaled the reduction accordingly. Figure $3 \mathrm{c}$ shows the relative emission level right after the pulse (averaged over 10 data points). As expected for stimulated emission, the effect occurs for wavelengths within the NV emission spectrum and is strongest around $700 \mathrm{~nm}$, which corresponds to the peak of the phonon sideband.

To understand the temporal dynamics, we modelled the NV by a simple three-state system (Fig. 1a). This model neglects the spin dynamics but is a good approximation since the green CW laser spin-polarizes into the $m_{\mathrm{s}}=0$ state and all emission processes are spin-conserving. The differential equations for the system probabilities $P_{j}$ to be in the states $|j\rangle$ in Fig. 1a are:

$$
\begin{gathered}
\dot{P}_{1}=-\Lambda_{\text {green }} P_{1}+L_{21} P_{2}+L_{31} P_{3} \\
\dot{P}_{2}=\Lambda_{\text {green }} P_{1}-\left[L_{21}+L_{23}+\Lambda_{\text {red }}(t)\right] P_{2}+\Lambda_{\text {red }}(t) P_{3} \\
\dot{P}_{3}=\left[L_{23}+\Lambda_{\text {red }}(t)\right] P_{2}-\left[L_{31}+\Lambda_{\text {red }}(t)\right] P_{3}
\end{gathered}
$$

where the pulsed laser created a stimulated emission rate $\Lambda_{\text {red }}(t)$ with a temporal Gaussian profile with standard deviation $6 \mathrm{ps}$, repetition rate of $(25 \mathrm{~ns})^{-1}=40 \mathrm{MHz}$ and overall strength is fitted to match the experiment. When we give time-independent values for $\Lambda_{\text {red }}$ below, we give the equivalent rate that a 6 ps square pulse would have. The spontaneous emission rate to $|3\rangle$ was taken to be $L_{23}=18$ $\mathrm{MHz}$ from ref. 34. All spontaneous emission rates to states other than $|3\rangle$ were grouped into one rate of $L_{21}=65.3 \mathrm{MHz}$ directly from state $|2\rangle$ to state $|1\rangle$. The value was chosen to correspond to a total lifetime of $12 \mathrm{~ns}$ for the excited state. The phonon-decay rate was set to $L_{31}=1 \mathrm{THz}$ but the exact value is irrelevant provided it is fast compared to all other transition rates. Starting the system in state $|1\rangle$ with the constant pumping rate $\Lambda_{\text {green }}$ we time-evolved the system via numerical integration until it reached a steady state; we then included the time-dependent repeating red pulses in the evolution.

In the simulations without the red pulses, the steady state has a given population in the excited state. Including the red pulses leads to stimulated emission on the $|2\rangle$ to $|3\rangle$ transition over the 6 ps pulse duration. This depletes population from the excited state to $|3\rangle$, which then decays to $|1\rangle$ due to the fast phononic decay. In the $25 \mathrm{~ns}$ between the pulses, the population of the excited state slowly 
a

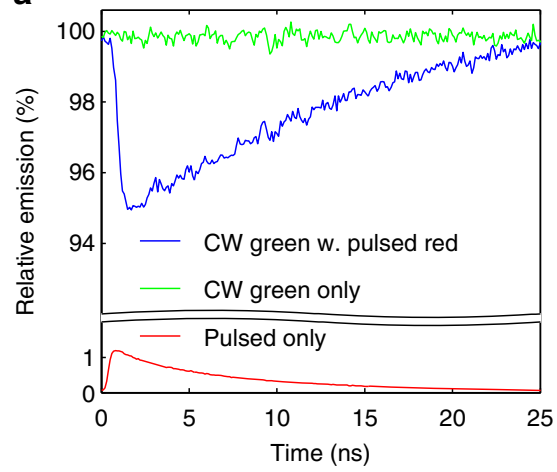

b

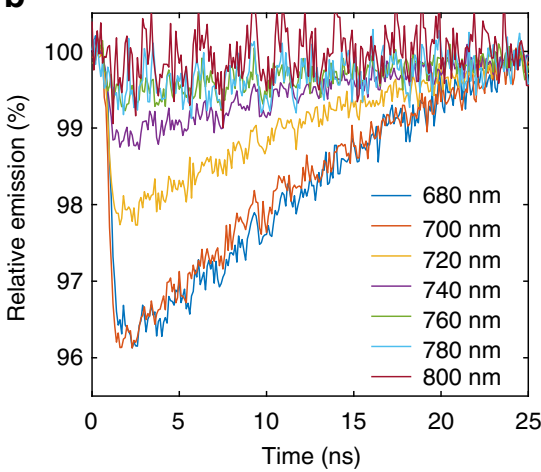

C

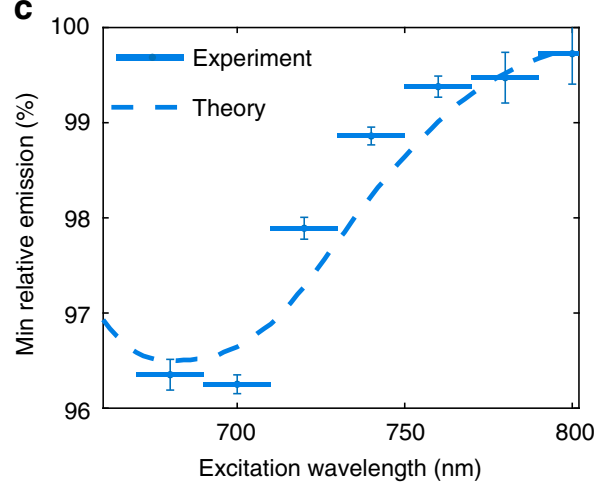

Figure 3 | Temporal emission reduction for different stimulating wavelengths. (a): raw data of temporally resolved emission which is spectrally integrated below $650 \mathrm{~nm}$. The fluorescence reduction occurs instantly at the point in time of the red pulse. Then the emission recovers in between pulses. (b) Temporal emission curves for different pulsed excitation wavelengths (c) The minimal relative emission directly after the pulse as a function of the pulsed excitation wavelength shows that the greatest reduction in emission occurs around $700 \mathrm{~nm}$ corresponding to the peak of the phonon sideband of the NV. The error bars give the standard deviation of the temporal data points averaged for each value.

recovers to its original value due to the constant green pumping rate. The number of spontaneously emitted photons also drops during the pulse duration and then recovers in between pulses because it is proportional to the excited state population. The timescale of the recovery is roughly the time between pulses, that is, $25 \mathrm{~ns}$, and decreases (that is, the recovery rate increases) with increasing green CW pump power.

To quantify the expected wavelength dependence for the effect for stimulated emission, we modelled different wavelengths of the pulsed laser by varying the stimulated emission cross section proportional to the measured NV emission spectrum with a maximum of $\Lambda_{\text {red }}=6$ $\mathrm{GHz}$ at $682 \mathrm{~nm}$. The pump rate was set to $\Lambda_{\text {green }}=92 \mathrm{MHz}$ and Fig. $3 \mathrm{c}$ shows that the resulting theoretical wavelength dependence of the relative emission matches qualitatively with the experimental measurements. We note that a transition rate of order $\mathrm{GHz}$ is realistic for a pulsed source with powers of few $\mathrm{W}$ during the $6 \mathrm{ps}$ pulse, and a focus-area on the order $1 \mu \mathrm{m}^{2}$.

The wavelength dependence is further evidence for stimulated emission as opposed to ionization. Ionization of the NV centre has been explained as an absorption process from the excited state to the conduction band. The absorption to the conduction band should therefore not show any wavelength dependence since the band can accommodate different photon energies. There should only be a sharp drop of the effect once the photon energy becomes too small (that is, wavelength too long) to reach the conduction band. Ionization should furthermore be more likely with increased excited state population, that is, increased absorption. If the emission reduction was caused by ionization then the wavelength dependence would follow the absorption spectrum of the $\mathrm{NV}$, which has its phonon sideband and maximum in wavelength range around $550 \mathrm{~nm}$ (below the ZPL) and not around $700 \mathrm{~nm}$ (above the ZPL) ${ }^{25}$.

While the pulsed laser wavelength changes the strength of the emission reduction, it does not seem to change the timescale of the recovery in Fig. 3b. This behaviour matches our simulations. The recovery of the emission in between pulses is approximately exponential. In the case that the effect is caused by stimulated emission the recovery should be purely caused by the green CW pump. For other effects, such as ionization, the recovery could be either explained with the crystal returning to its equilibrium state, with a rate set by internal NV or crystal parameters, which should be slower and independent of the green pump rate. For ionization, the recovery could also be explained by the green laser ionizing back to the $\mathrm{NV}^{-}$state, which has previously been measured to occur at a rate of $(0.25 \mu \mathrm{s})^{-1}$ for a $532 \mathrm{~nm}$ laser at
$0.25 \mathrm{~mW}$ power (see Fig. 3c in ref. 26), which is an order of magnitude slower than the rate we find.

Figure 4 a shows a measurement of the emission dynamics with varying powers of the green $\mathrm{CW}$ pump power. The pulse repetition rate was reduced to $(100 \mathrm{~ns})^{-1}=10 \mathrm{MHz}$ to ensure that the system has enough time to return to its equilibrium state between pulses. Since stronger green CW pumping leads to higher total emission, we plot the relative emission as a percentage of the highest total emission respectively. We observed a shorter recovery time, i.e., faster recovery rate, for stronger green CW power: in Fig. 4a, the lowest power (pink curve) has the slowest recovery rate. With increasing green power (light blue, orange, dark blue), the emission recovers faster and the highest power (green) shows the fastest recovery rate. The recovery time for $0.25 \mathrm{~mW}$ (pink) is $\sim 25 \mathrm{~ns}$, which is much faster than the expected $250 \mathrm{~ns}$ for ionization ${ }^{26}$. The recovery timescales are consistent with stimulated emission as demonstrated below. For lower green powers, the relative emission reduces to only $\sim 97 \%$ (pink) rather than $\sim 93 \%$ (green and dark blue). This is explained by the fact that the red pulsed laser weakly excites the NV as demonstrated below.

The observed dynamics was reproduced by our simulations: corresponding curves are plotted in the same colours in Fig. 4a,b. The stimulated emission rate was set to $\Lambda_{\text {red }}=13 \mathrm{GHz}$ to reproduce the relative emission reduction to $\sim 93 \%$ for large powers. The pump rates were varied proportional to the green laser powers in the experiment from 8.8 to $141 \mathrm{MHz}$, which corresponds to an average focus area of $(0.6 \mu \mathrm{m})^{2}$, given the known absorption cross-section $0.95 \times 10^{-16} \mathrm{~cm}^{2}$ (ref. 35). These pump rates lead to recovery timescales similar to the experiment both in absolute values and relative trend between the curves. To reproduce the fact that lower green powers show a weaker emission reduction effect, we included an excitation rate of $\Lambda_{\text {red2 }}=0.85 \mathrm{GHz}$ due to the red pulse from ground to excited state:

$$
\begin{gathered}
\dot{P}_{1}=-\left[\Lambda_{\text {green }}+\Lambda_{\text {red } 2}(t)\right] P_{1}+L_{21} P_{2}+L_{31} P_{3} \\
\dot{P}_{2}=\left[\Lambda_{\text {green }}+\Lambda_{\text {red } 2}(t)\right] P_{1}-\left[L_{21}+L_{23}+\Lambda_{\text {red }}(t)\right] P_{2} \\
+\Lambda_{\text {red }}(t) P_{3} \\
\dot{P}_{3}=\left[L_{23}+\Lambda_{\text {red }}(t)\right] P_{2}-\left[L_{31}+\Lambda_{\text {red }}(t)\right] P_{3}
\end{gathered}
$$

This rate $\Lambda_{\text {red2 }}$ was calculated using the weak effective absorption cross-section for $\mathrm{NV}^{-}$at $700 \mathrm{~nm}$, which we measured 
to be $3 \times 10^{-2} \mathrm{~m}^{24}$. We had initially neglected this as it is only a weak effect but we found that for low green powers (which resulted in weak total emission) the inclusion of this effect in the modelling was a good explanation for why the pink curve only reduces to $\sim 97 \%$ while higher green powers reduce to $\sim 93 \%$.

All measurements so far were taken with single crystal diamond. We compared a single crystal sample to nanodiamonds on a silicon substrate under the same conditions. The same effects were observed and confirmed the generality of our results, see Supplementary Note 1 .

Direct measurement of stimulated emission. As final confirmation that the emission reduction is caused by stimulated emission we have measured the stimulated emission at the stimulating wavelength directly. To measure the stimulated emission signal against the much stronger red laser signal and its associated noise, we have modulated the green pump laser with a frequency of $548 \mathrm{~Hz}$ and changed the red laser from a pulsed to a CW source. For details of the setup, see Fig. 5a and the 'Methods' section. Thus, a stimulated emission signal due to the green pump laser must also be modulated, while the red laser signal is not. A lock-in amplifier allowed us to filter only the frequency of interest and thus removed the red laser signal and a majority of the noise. Figure 5b shows that the resulting signal is indeed almost zero when only the red CW laser is on. When only the modulated green pump is on some spontaneous emission is produced at the modulation frequency but we block most of that out with a $705 \mathrm{~nm}$ bandpass filter to see only stimulated emission. When the red CW laser is added to the green pump laser, Fig. 5 shows that the emission signal increases linearly with the red stimulating laser power. This increase is only measured because it oscillates at the modulation frequency of the green pump and is thus clearly stimulated emission because it is pumped with the green laser and emitted due to the red laser. This is the first direct measurement of stimulated emission from the NV centre. We note that the spontaneous emission signal from only the green laser is negligible compared with the stimulated emission signal for two reasons: We used a bandpass filter around the red laser wavelength and thus removed the majority of spontaneous emission and additionally the spontaneous emission is isotropic and only the fraction of the solid angle of the collecting objective is detected whereas the stimulated emission is directional in the stimulating beam direction and therefore has very high collection efficiency. This leads to the given configuration with excellent contrast between the stimulated emission signal and the spontaneous emission from the green laser. For higher green powers, the spontaneous emission signal strengthens while the detection

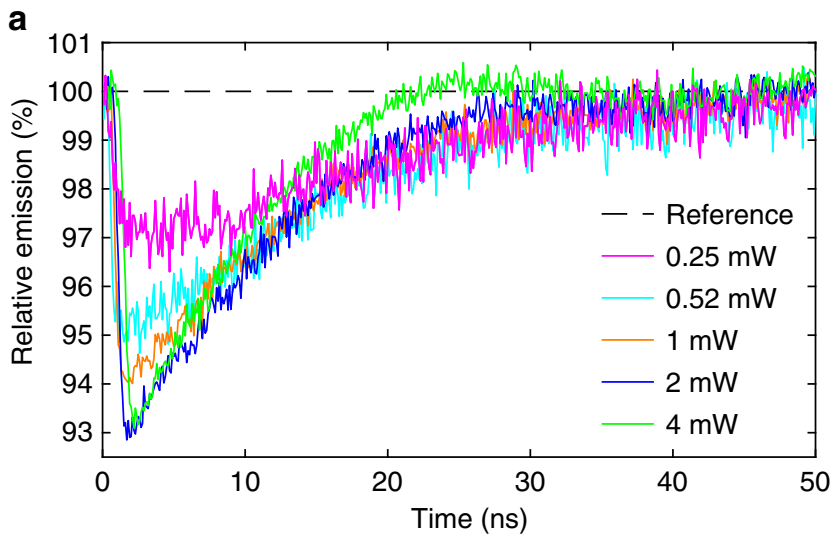

contrast is not as strong. This measurement was performed in transmission through the sample using an objective on each side, see Fig. 5a.

\section{Discussion}

We have shown clear signatures of stimulated emission in $\mathrm{NV}$ centres in single crystal diamond as well as nanodiamonds. We demonstrated the spontaneous NV emission from a green CW pump laser and the reduction of this spontaneous emission due to a strong pulsed red laser at the centre of the phonon sideband $(\sim 700 \mathrm{~nm})$. We explain this reduction in spontaneous emission as being due to stimulated emission at $700 \mathrm{~nm}$. The measured emission reduction follows all the expected behaviour for stimulated emission in terms of its dependence on the wavelength of the pulsed laser, the spectral reduction of both the $\mathrm{NV}^{-}$and the $\mathrm{NV}^{0} \mathrm{ZPL}$, as well as the temporal dynamics, including emission reduction during the laser pulse and a recovery rate that varies with the green laser power. Our theoretical modelling of stimulated emission also reproduces the measured effects.

Particularly we show that for wavelengths $\sim 700 \mathrm{~nm}$ stimulated emission is the dominant process over ionization. This is indicated by no increase of either ZPL line, a wavelength dependence, which follows the emission rather than the absorption spectrum and a temporal dynamics inconsistent with ionization. We furthermore showed the transition to the regime where ionization dominates, which is when the pulsed laser is set to wavelengths around the $\mathrm{NV}^{-} \mathrm{ZPL}$. Our results are therefore consistent with previously reported photoionization studies $\mathrm{NV}^{-} \leftrightarrow \mathrm{NV}^{0}$ induced by wavelengths below $650 \mathrm{~nm}$.

Our findings manifest that stimulated emission dominating over ionization is dependent on the wavelength (rather than the intensity), which should be in the phonon sideband $\lambda>650 \mathrm{~nm}$. This is in agreement with previous literature on super-resolution microscopy, where stimulated emission depletion was achieved with wavelengths $\lambda>700 \mathrm{~nm}$ pulses ${ }^{36-38}$, whereas charge state depletion was achieved with $637 \mathrm{~nm}$ pulses ${ }^{39,40}$.

The measurement of the effect in both single-crystal diamond and nanodiamonds shows sample-independent applicability.

Our results show that stimulated emission can be utilized in the $\mathrm{NV}$ and lead the way to exploring the benefits of stimulated emission, for example, for higher collection efficiency into a cavity mode. Our results also indicate that NV centres are suitable as a laser medium. This opens up new avenues of applications for the NV centre.

We estimate the conditions for lasing with NV centres for the three-phonon sideband transition at $709 \mathrm{~nm}$, where stimulated emission strongly dominates. Lasing is possible, when the rate of

\section{b}

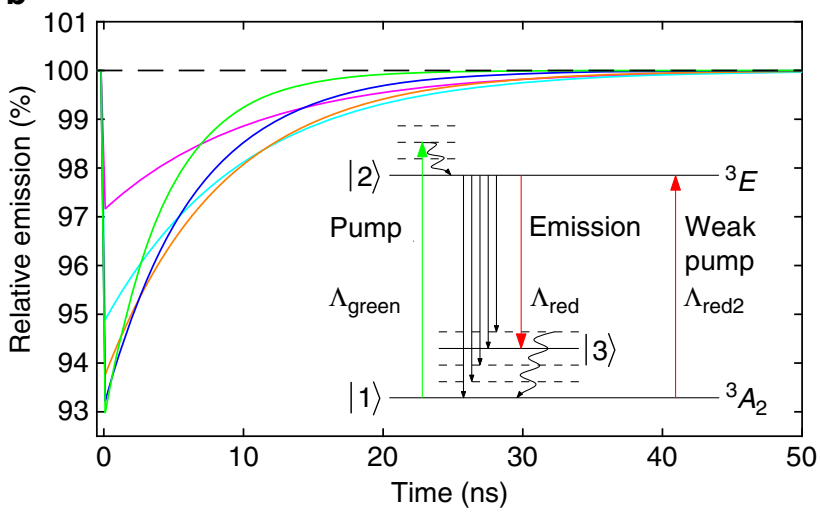

Figure 4 | Emission reduction and recovery for different green $\mathbf{C W}$ powers. (a) Experimental data where the red pulse was at $t \approx 1 \mathrm{~ns}$. The recovery rate increases with increasing green power. The time-averaged red power was measured as $1.73 \mathrm{~mW}$. (b) The theoretical modelling, reproducing a. 
a

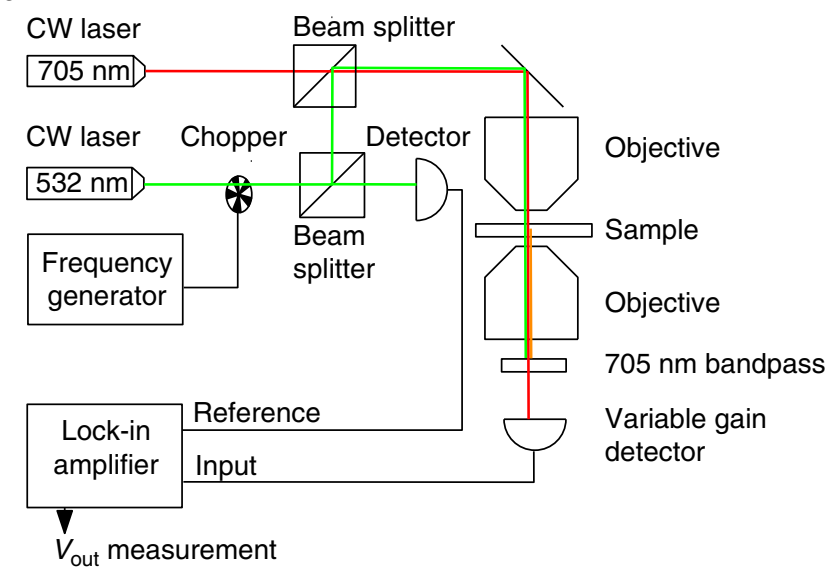

b

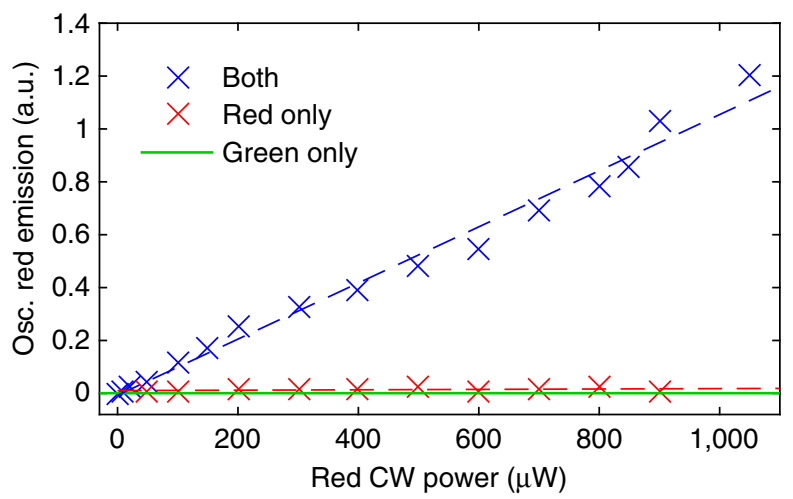

Figure 5 | Direct measurement of stimulated emission. (a) Setup with modulation on the green laser and a lock-in amplifier filtering the detection signal for only the modulation frequency (b) The red CW laser creates stimulated emission. Because the stimulated emission is dependent on the green pump laser, it also oscillates at the modulation frequency and is hence detected. The red CW laser signal itself is unmodulated and is thus filtered out by the lock-in amplifier. The green laser by itself creates fluorescence, which is almost entirely filtered out by the $705 \mathrm{~nm}$ bandpass and additionally only the fraction that is emitted into the solid angle of the objective is directed to the detector. The stimulated emission is emitted into the laser beam direction and is thus entirely collected by the detector and gives a clear signal linear with stimulating power. The blue and red dashed lines are linear fits.

stimulated emission into an optical cavity is greater than the loss rate $\kappa$ of the cavity. The stimulated emission rate can be calculated $^{41,42}$ on the basis of the cavity and spontaneous emission characteristics. From this condition, we find the minimum density of NV centres to achieve lasing:

$$
n_{\mathrm{NV}}=\frac{4 \pi^{3} c \Delta v_{23} n^{3} \frac{V_{\mathrm{cav}}}{V_{\text {diam }}}}{3 v_{23} L_{23} \lambda^{3} n l_{\text {cav }}}=\frac{V_{\text {cav }}}{V_{\text {diam }}} \frac{3.6 \text { p.p.m. }}{\mathcal{F}\left(l_{\text {cav }} \text { in mm }\right)}
$$

where $c$ is the speed of light, $n=2.4$ the refractive index of diamond, $\lambda=709 \mathrm{~nm}$ the free-space wavelength of the threephonon sideband, $v_{23}=c / \lambda$ the corresponding frequency, $\Delta v_{23} \approx 24 \mathrm{THz}$ the corresponding width of the three-phonon peak $^{15,43}, l_{\text {cav }}$ the cavity length, $\mathcal{F}$ the cavity finesse, $V_{\text {cav }}$ the cavity mode volume and $V_{\text {diam }}$ the diamond volume within the cavity mode. From this equation, we see that the required density reduces proportional to $1 /$ finesse and that lasing is in principle possible with a commonly achieved density of 0.1 to 1 p.p.m. even for low-finesse cavities. However, low-finesse cavities will require very large pump powers for lasing. The required power at the lasing threshold can be estimated by the absorption cross-section and enforcing the detailed balance condition for the excited and ground state population $\rho_{\text {exd }} / \rho_{\mathrm{gr}}=\Gamma / \Lambda$ with pumping rate $\Lambda$ and emission rate $\Gamma$ at threshold, which is valid for not too small NV numbers in the cavity. We obtain

$$
P_{\text {thresh }}=\frac{1.2 \times 10^{4} \mathrm{~W}\left(A_{\text {cav }} \text { in } \mathrm{mm}^{2}\right)}{\mathcal{F}\left(l_{\text {cav }} \text { in mm }\right)\left(n_{\mathrm{N} V} \text { in p.p.m. }\right) \frac{V_{\text {diam }}}{V_{\text {cav }}}}
$$

This shows that while lasing is challenging, it should be possible, for example, for a cavity of $1 \mathrm{~mm}$ length with finesse $\mathcal{F}=10^{5}$ and average illumination area $A_{\text {cav }}=1 \mathrm{~mm}^{2}$ and an illuminated diamond volume that is half the cavity volume with an NV concentration $[\mathrm{NV}]=1$ p.p.m. requiring a pump power of $240 \mathrm{~mW}$. We confirmed numerically that this analytical approximation gives a good estimate for the required power by solving numerically the full seven-state model ${ }^{1}$ of the NV centre coupled to a laser cavity ${ }^{42}$.

Laser threshold magnetometry ${ }^{42}$ was recently proposed as a novel way of measuring magnetic fields with NV centre ensembles, enabling higher contrast and stronger output signals using stimulated emission. By driving an $\mathrm{NV}$ laser at the threshold, sensitivities down to fT $\mathrm{Hz}^{-1 / 2}$ could be achieved and could enable a robust, room-temperature alternative for SQUID sensors. The current results support the feasibility of the laser threshold sensing scheme.

\section{Methods}

Diamond sample preparation. We confirmed the effects on different diamond samples: sample A was a single crystal, type $1 \mathrm{~b}$, irradiated with $2 \mathrm{MeV}$ electrons to $2 \times 10^{18} \mathrm{e} \mathrm{cm}^{-2}$, annealed at $800^{\circ} \mathrm{C}$ for $5 \mathrm{~h}$. Sample B was a single crystal, type $1 \mathrm{~b}$, irradiated with $2 \mathrm{MeV}$ electrons to a fluence of $1.1 \times 10^{18} \mathrm{e} \mathrm{cm}^{-2}$ and annealed in vacuum at $900^{\circ} \mathrm{C}$ for $2 \mathrm{~h}$. During irradiation, the sample was in a dry nitrogen environment and kept at temperature below $800^{\circ} \mathrm{C}$ to avoid any surface degradation. Sample $\mathrm{C}$ was a single crystal, type $1 \mathrm{~b}$, irradiated with $2 \mathrm{MeV}$ electrons to a fluence of $1 \times 10^{18} \mathrm{e} \mathrm{cm}^{-2}$ and annealed in vacuum at $800^{\circ} \mathrm{C}$ for $2 \mathrm{~h}$. Sample C was $50 \mu \mathrm{m}$ thin used specifically for Fig. 5 to fit between the two objectives. Sample D were nanodiamonds, see Supplementary Note 1,irradiated with $2 \mathrm{MeV}$ electrons to a fluence of $1 \times 10^{18} \mathrm{e} \mathrm{cm}^{-2}$, vacuum annealed at $800{ }^{\circ} \mathrm{C}$ for $2 \mathrm{~h}$, then oxydized in air at $510^{\circ} \mathrm{C}$ for $4 \mathrm{~h}$.

Lasing conditions calculations. To obtain an analytical estimate for the required pump power $P_{\text {thresh }}$, connected to the pump rate $\Lambda_{\text {thresh }}=P_{\text {thresh }} \sigma /\left(A_{\text {cav }} \hbar \omega\right)$ via the known absorption cross-section $\sigma$ and the photon energy $\hbar \omega$, we enforced the detailed balance, that is, the ratio of transition rates equal the ratio of populations for the ground and excited state $\rho_{\text {exc }} / \rho_{\text {gr }}=\Gamma / \Lambda$ from which we obtain the excited state population $\rho_{\mathrm{exc}}=\Lambda /(\Gamma+\Lambda) \approx \Lambda / \Gamma$ for a high enough number of NV centres such that $\rho_{\mathrm{gr}} \gg \rho_{\mathrm{exc}} \Rightarrow \Gamma \gg \Lambda$ at threshold, which we confirmed numerically for $n_{\mathrm{NV}} \geq 0.01$ p.p.m. $\times\left(l_{\text {cav }}\right.$ in mm $)$. Combining the detailed balance condition with the lasing condition $G \rho_{\mathrm{exc}}=\kappa$ for the coupling $G$ of NV centres and cavity and cavity loss rate $\kappa$, we find the required pump rate for lasing $\Lambda_{\text {thresh }}=\Gamma \kappa / G$ from which we can then calculate the required power $P_{\text {thresh. }}$. We note that the expression becomes invalid when the absorbed power under homogeneous illumination $P_{\text {abs }}=n_{\mathrm{NV}} n_{\mathrm{C}} V_{\text {cav }} \Lambda \hbar \omega>P_{\text {thresh }}$, since the laser medium becomes strongly absorbing in this case. Here $n_{\mathrm{C}}$ is the carbon density of diamond. This can be easily monitored and happens for very long cavity designs and very high NV densities.

Setup for direct measurement of stimulated emission. For the direct measurement of stimulated emission, we modulated the green $532 \mathrm{~nm}$ laser with an optical chopper at a frequency of $548 \mathrm{~Hz}$ and combined this via a fibre beam splitter with a CW $705 \mathrm{~nm}$ red laser. Since the stimulated emission is directional in the stimulating beam direction, we measured in transition using one objective $(\times 10, \mathrm{NA}=0.25)$ to focus the beam inside the sample and one objective $(\times 40$ $\mathrm{NA}=0.65)$ to collect the light after passing the sample once. Owing to the short working distance of the lenses, we used a thin sample of only $50 \mu \mathrm{m}$ thickness to ensure the ability to focus both objectives to the same point in the sample and collimate the outgoing beam. The signal was detected via a variable gain detector and then frequency filtered with a lock-in amplifier using the chopper frequency as a reference. Thus we ensured that only emission caused by the green pump laser was detected. Since the spontaneous emission is isotropic only the fraction that goes into the solid angle of the collecting lens is detected. The directional stimulated emission is collected entirely when the outcoming laser beam is collimated by the collecting objective. 
Data availability. The data that support the findings of this study are available from the corresponding author on request.

\section{References}

1. Doherty, M. W. et al. The nitrogen-vacancy colour centre in diamond. Phys. Rep. 528, 1-45 (2013).

2. Aharonovich, I. et al. Producing optimized ensembles of nitrogen-vacancy color centers for quantum information applications. J. Appl. Phys. 106, 124904 (2009).

3. Neumann, P. et al. Quantum register based on coupled electron spins in a room-temperature solid. Nat. Phys. 6, 249-253 (2010).

4. Henderson, M. R. et al. Diamond in tellurite glass: a new medium for quantum information. Adv. Mater. 23, 2806-2810 (2011).

5. Schirhagl, R., Chang, K., Loretz, M. \& Degen, C. L. Nitrogen-vacancy centers in diamond: nanoscale sensors for physics and biology. Annu. Rev. Phys. Chem. 65, 83-105 (2014)

6. Balasubramanian, G. et al. Nanoscale imaging magnetometry with diamond spins under ambient conditions. Nature 455, 648-651 (2008).

7. Maze, J. R. et al. Nanoscale magnetic sensing with an individual electronic spin in diamond. Nature 455, 644-647 (2008).

8. Fang, K. et al. High-sensitivity magnetometry based on quantum beats in diamond nitrogen-vacancy centers. Phys. Rev. Lett. 110, 130802 (2013).

9. Grinolds, M. S. et al. Nanoscale magnetic imaging of a single electron spin under ambient conditions. Nat. Phys. 9, 215-219 (2013).

10. Jensen, K. et al. Cavity-enhanced room-temperature magnetometry using absorption by nitrogen-vacancy centers in diamond. Phys. Rev. Lett. 112, 160802 (2014).

11. Rondin, L. et al. Magnetometry with nitrogen-vacancy defects in diamond. Rep. Prog. Phys. 77, 056503 (2014).

12. Dolde, F. et al. Electric-field sensing using single diamond spins. Nat. Phys. 7, 459-463 (2011).

13. Dolde, F. et al. Nanoscale detection of a single fundamental charge in ambient conditions using the $\mathrm{NV}^{-}$center in diamond. Phys. Rev. Lett. 112, 097603 (2014).

14. Neumann, P. et al. High-precision nanoscale temperature sensing using single defects in diamond. Nano Lett. 13, 2738-2742 (2013).

15. Plakhotnik, T., Doherty, M. W., Cole, J. H., Chapman, R. \& Manson, N. B. Alloptical thermometry and thermal properties of the optically detected spin resonances of the $\mathrm{NV}^{-}$center in nanodiamond. Nano Lett. 14, 4989-4996 (2014).

16. Doherty, M. W. et al. Electronic properties and metrology applications of the diamond $\mathrm{NV}^{-}$center under pressure. Phys. Rev. Lett. 112, 047601 (2014).

17. McGuinness, L. P. et al. Quantum measurement and orientation tracking of fluorescent nanodiamonds inside living cells. Nat. Nanotechnol. 6, 358-363 (2011).

18. Kucsko, G. et al. Nanometre-scale thermometry in a living cell. Nature 500, 54-59 (2013).

19. Clevenson, H. et al. Broadband magnetometry and temperature sensing with a light-trapping diamond waveguide. Nat. Phys. 11, 393-397 (2015).

20. Mildren, R. P. \& Sabella, A. Highly efficient diamond raman laser. Opt. Lett. 34, 2811-2813 (2009).

21. Rand, S. C. in Tunable Solid State Lasers II. Vol. 52 of Springer Series in Optical Sciences 276 (Springer, 1986).

22. Faraon, A., Barclay, P. E., Santori, C., Fu, K.-M. C. \& Beausoleil, R. G. Resonant enhancement of the zero-phonon emission from a colour centre in a diamond cavity. Nat. Photonics 5, 301-305 (2011).

23. Rittweger, E., Han, K. Y., Irvine, S. E., Eggeling, C. \& Hell, S. W. Nat. Photonics 3, 144-147 (2009).

24. Laporte, G. \& Psaltis, D. STED imaging of green fluorescent nanodiamonds containing nitrogen-vacancy-nitrogen centers. Biomed. Opt. Express 7, 34-44 (2016).

25. Manson, N. \& Harrison, J. Photo-ionization of the nitrogen-vacancy center in diamond. Diam. Relat. Mater. 14, 1705-1710 (2005).

26. Waldherr, G. et al. Dark states of single nitrogen-vacancy centers in diamond unraveled by single shot NMR. Phys. Rev. Lett. 106, 157601 (2011).

27. Beha, K., Batalov, A., Manson, N. B., Bratschitsch, R. \& Leitenstorfer, A. Optimum photoluminescence excitation and recharging cycle of single nitrogen-vacancy centers in ultrapure diamond. Phys. Rev. Lett. 109, 097404 (2012).

28. Aslam, N., Waldherr, G., Neumann, P., Jelezko, F. \& Wrachtrup, J. Photo-induced ionization dynamics of the nitrogen vacancy defect in diamond investigated by single-shot charge state detection. New. J. Phys. 15, 013064 (2013).

29. Siyushev, P. et al. Optically controlled switching of the charge state of a single nitrogen-vacancy center in diamond at cryogenic temperatures. Phys. Rev. Lett. 110, 167402 (2013)

30. Chen, X.-D., Zou, C.-L., Sun, F.-W. \& Guo, G.-C. Optical manipulation of the charge state of nitrogen-vacancy center in diamond. Appl. Phys. Lett. 103, 013112 (2013)
31. Shields, B. J., Unterreithmeier, Q. P., de Leon, N. P., Park, H. \& Lukin, M. D. Efficient readout of a single spin state in diamond via spin-to-charge conversion. Phys. Rev. Lett. 114, 136402 (2015).

32. Davies, G. \& Hamer, M. F. Optical studies of the $1.945 \mathrm{ev}$ vibronic band in diamond. Proc. R. Soc. Lond. A 348, 285-298 (1976).

33. Su, C.-H., Greentree, A. D. \& Hollenberg, L. C. L. Towards a picosecond transform-limited nitrogen-vacancy based single photon source. Opt. Express 16, 6240-6250 (2008).

34. Su, C.-H. Novel Quantum Technology Based on Atom-Cavity Physics. (PhD thesis Univ. Melbourne, 2010).

35. Chapman, R. \& Plakhotnik, T. Quantitative luminescence microscopy on nitrogen-vacancy centres in diamond: Saturation effects under pulsed excitation. Chem. Phys. Lett. 507, 190-194 (2011).

36. Han, K. Y. et al. Three-dimensional stimulated emission depletion microscopy of nitrogen-vacancy centers in diamond using continuous-wave light. Nano Lett. 9, 3323-3329 (2009).

37. Wildanger, D., Maze, J. R. \& Hell, S. W. Diffraction unlimited all-optical recording of electron spin resonances. Phys. Rev. Lett. 107, 017601 (2011).

38. Arroyo-Camejo, S. et al. Stimulated emission depletion microscopy resolves individual nitrogen vacancy centers in diamond nanocrystals. ACS Nano 7, 10912-10919 (2013)

39. Han, K. Y., Kim, S. K., Eggeling, C. \& Hell, S. W. Metastable dark states enable ground state depletion microscopy of nitrogen vacancy centers in diamond with diffraction-unlimited resolution. Nano Lett. 10, 3199-3203 (2010).

40. Chen, X. et al. Subdiffraction optical manipulation of the charge state of nitrogen vacancy center in diamond. Light Sci. Appl. 4, e230 (2015).

41. Siegman, A. E. Lasers (Univ. Science Books, 1986).

42. Jeske, J., Cole, J. H. \& Greentree, A. D. Laser threshold magnetometry. New. J. Phys. 18, 013015 (2016).

43. Kehayias, P. et al. Infrared absorption band and vibronic structure of the nitrogen-vacancy center in diamond. Phys. Rev. B 88, 165202 (2013).

\section{Acknowledgements}

We thank Jason Twamley, Neil Manson and Audrius Alkauskas for useful discussions and comments. This work was supported by the Australian Research Council under the Discovery (ARC DP130104381), LIEF (LE140100131), Centres of Excellence (CE110001013 and CE140100003) and Future Fellowship (FT110100225) schemes.

\section{Author contributions}

JJ, DWML, LPM, PR, JHC, BCG, ADG, XV and TV conceived and planned the experiments. JJ, DWML, XV, LPM and PR carried out the experiments. JJ, ADG and JHC planned and carried out the simulations. SO, TO, JJ, DWML, PR, LPM, FJ, BCJ and JCM contributed to sample preparation. JJ, DWML, XV, LPM, PR, MWD, FJ, TV, JHC, BCG and ADG contributed to the interpretation of the results. JJ took the lead in writing the manuscript. All authors provided critical feedback and helped shape the research, analysis and manuscript.

\section{Additional information}

Supplementary Information accompanies this paper at http://www.nature.com/ naturecommunications

Competing financial interests: The authors declare no competing financial interests.

Reprints and permission information is available online at http://npg.nature.com/ reprintsandpermissions/

How to cite this article: Jeske, J. et al. Stimulated emission from nitrogen-vacancy centres in diamond. Nat. Commun. 8, 14000 doi: 10.1038/ncomms14000 (2017).

Publisher's note: Springer Nature remains neutral with regard to jurisdictional claims in published maps and institutional affiliations.

(c) (1) (2) This work is licensed under a Creative Commons AttributionNonCommercial-ShareAlike 4.0 International License. The images or other third party material in this article are included in the article's Creative Commons license, unless indicated otherwise in the credit line; if the material is not included under the Creative Commons license, users will need to obtain permission from the license holder to reproduce the material. To view a copy of this license, visit http:// creativecommons.org/licenses/by-nc-sa/4.0/

(C) The Author(s) 2017 